Bull. Austral. Math. Soc.

VOL. 56 (1997) [243-251]

\title{
A CHARACTERISATION OF CYCLIC SUBNORMAL SEPARATED $A$-GROUPS OF NILPOTENT LENGTH THREE
}

\author{
MUHAMmad UMAR MíaKarfi
}

The paper gives a detailed description of all those finite A-groups of nilpotent length three that satisfy the cyclic subnormal separation condition. It is shown that every monolithic group under discussion is an extension of its Fitting subgroup $P$, which is a homocyclic $p$-group, by a $p^{\prime}$ metabelian subgroup $H$, where $p$ is a prime. The centraliser of $P$ in $H$ is trivial while the monolith $W$ is equal to $\Omega_{1}(P)$ and the action of $H$ on $W$ is faithful and irreducible. $H$ is further shown to have non trivial centre and is an extension of its derived subgroup $M$ by a subgroup $L$ such that

$$
[M, L]=M \text { and }\left[M_{q}, L_{q}\right]=1
$$

for all primes $q$, where $M_{q}$ and $L_{q}$ are the respective Sylow $q$-subgroups of $M$ and $L$. The Fitting subgroup $F$ of $H$ is shown to be $M \times Z(H)$, while $Z(H)=F \cap L$ and every element of $L$ of prime order is in $Z(H)$. Finally it is shown that if $q^{\ell(q)}$ is the exponent of $M_{q}$ then every element of order dividing $q^{\ell(q)}$ in $L$ belongs to $Z(H)$.

\section{INTRODUCTION}

Let $G$ be a finite group and $A, B$ cyclic subgroups of $G$ such that $B \leqslant A$. $G$ is said to be a $C S_{n}$ group if for any such pair of cyclic subgroups there exists a subnormal subgroup $N$ in $G$ such that $N \cap A=B$. We call this the cyclic subnormal separation condition. It has been shown in [2] that the class of $C S_{n}$ groups satisfy, among other closure properties, the subgroup, quotient group and finite direct product closure properties.

It was also shown [2] that any class, closed under the above three operations, consists precisely of the subdirect products of the monolithic groups in the class.

Thus in our discussion it is enough to consider only the monolithic groups. We discuss $A$-groups as a step towards the discussion on general soluble groups. In this paper we provide a fairly complete description of those $A$-groups of nilpotent length three in $C S_{n}$.

Received 30 October 1996

Copyright Clearance Centre, Inc. Serial-fee code: 0004-9729/97 \$A2.00+0.00. 
A group $G$ is said to be a $A$-group if it is soluble and every Sylow subgroup of $G$ is Abelian. A-groups present a very interesting class of soluble groups, they possess very nice properties. Detailed discussions on these properties can be found in [6].

The main result in the paper is presented in Theorem $A$ as follows:

Theorem A. Let $G$ be a monolithic A-group of nilpotent length three. Then $G$ is a $C S_{n}$ group if and only if

1. $G=P \rtimes H$ is a semidirect product of a normal $p$-subgroup $P$, for some prime $p$, with a $p^{\prime}$-metabelian subgroup $H$, where $P=F(G)$, the Fitting subgroup of $G$, is homocyclic and $C_{H}(P)=1$. Also the monolith $W=\Omega_{1}(P)$ and the action of $H$ on $W$ is faithful and irreducible.

2. (a) $H=M \rtimes L$ where $M=H^{\prime}$ is the derived subgroup of $H$ and if $M_{q}, L_{q}$ denote the Sylow $q$-subgroups of $M$ and $L$ respectively, then $\left[M_{q}, L_{q}\right]=$ 1 for all $q$.

(b) The centre $Z(H)$ of $H$ is non trivial, $L$ is cyclic and $[M, L]=M$.

(c) The Fitting subgroup $F$ of $H$ is given by $F=M \times Z(H)$. Also $Z(H)=$ $F \cap L$ and every element of $L$ of prime order is in $Z(H)$.

3. Let $q^{\ell(q)}$ be the exponent of $M_{q}$. Then for all $q$ every element of order dividing $q^{\ell(q)}$ in $L$ belongs to $Z(H)$.

\section{Preliminaries}

We collect in this section those results that we need to prove Theorem A. The first result gives the necessary and sufficient conditions for a group that is a split extension of a $p$-group, to be in $C S_{n}$.

Theorem 2.1. [2, Theorem A] Suppose that $P$ is a p-group where $p$ is a prime, and $H$ is a group acting on $P$. Let $G=P \rtimes H$ be the semidirect product of $P$ by $H$. Then $G$ is a $C S_{n}$ group if and only if

1. $H$ is a $C S_{n}$ group, and

2. $\left[P,\langle y\rangle^{\cdot H}\right] \cap C_{p}(y)=1$

for every $p^{\prime}$-element $y$ of $H$ of prime power order (where $C_{P}(y)$ is the centraliser of $y$ in $P$ ).

We use the notation $H^{\cdot \cdot G}$ to denote the subnormal closure of $H$ in $G$. This is the smallest subnormal subgroup of $G$ that contains $H$.

THEOREM 2.2. [1, Theorem 5.3.6] If $A$ is a $p^{\prime}$-group of automorphisms of the p-group $P$, for some prime $p$, then

$$
[P, A,]=[P, A]
$$


We sometimes refer to the above result as co-prime action. The next result lists some of the properties of $A$-groups, the proof of this and other general properties of $A$-groups can be found in [6].

Lemma 2.3. Let $G$ be an A-group, then

1. G splits over each subgroup in its derived series.

2. If $Z(G)$ and $G^{\prime}$ are the centre and the derived subgroup of $G$ respectively then

$$
G^{\prime} \cap Z(G)=1
$$

The following result can be found in [3, Section 4].

Lemma 2.4. Suppose that $G$ is a monolithic A-group. Then:

(a) The monolith $W$ is an elementary Abelian p-group for some prime $p$;

(b) The Fitting subgroup, $F=F(G)$, of $G$ is a homocyclic p-group.

(c) The centraliser of $W$ in $G$ is exactly $F$.

(d) $G=F \rtimes H$ for some $p^{\prime}$-subgroup $H$ of $G$.

Recall that a monolithic group is a group that has a unique minimal normal subgroup and the unique minimal normal subgroup is called the monolith. We have another result on monolithic $A$-groups, this is [4, Lemma 2.3].

Lemma 2.5. Let $G$ be a monolithic A-group with monolith $W$ which is a $p$ group for some prime $p$. If $G$ is in $C S_{n}$ then it has a $p^{\prime}$-subgroup $H$ such that

$$
G=P \rtimes H
$$

for some p-subgroup $P$ and the Fitting subgroup $F(H)$ of $H$ contains all the elements of $H$ of prime order.

The next result gives a necessary and sufficient condition for an $A$-group, which is an extension of a certain $p$-subgroup by a $p^{\prime}$-subgroup, to be in $C S_{n}$.

Proposition 2.6. [3, Theorem C] Suppose that $G=W \times H$ is an A-group with $W$ a minimal normal p-subgroup for some prime $p$ and $H$ a metabelian $p^{\prime}$ subgroup acting faithfully on $W$. Then $G$ is a $C S_{n}$ group if and only if every element of $H \backslash F(H)$ acts fixed point freely on $W$.

The next result shows that every metabelian group is a $C S_{n}$ group.

LEMma 2.7. [2, Lemma 3.5] If $G$ is metabelian then $G$ is in $C S_{n}$.

Theorem 2.8. Suppose that $P$ is an elementary Abelian p-group, $H$ a homocyclic $q$-group of exponent $q^{\ell}$ which is a subgroup of Aut $P$, where $p$ and $q$ are distinct 
primes. Suppose also that $H$ is non cyclic. Then $P$ is generated by the centralisers of the cyclic subgroups of $H$ of order $q^{\ell}$.

PRoOF: We know that by $[1,3.3 .3]$

$$
P=\left\langle G_{p}(h), h \in H^{+}\right\rangle
$$

where $H^{+}$denotes the non-trivial elements of $H$. Also as an $F_{P} H$-module

$$
P=\bigoplus_{i=1}^{s} P_{i}
$$

where each $P_{i}$ is an irreducible $H$-module. Since $H$ is Abelian

$$
C_{P_{i}}(h)=0 \quad \text { or } \quad P_{i} \quad \forall h \in H^{+} .
$$

Now to get the result it is enough to show that

$$
L_{i}=\left\langle h \in H, C_{P_{i}}(h) \neq 0\right\rangle=C_{H}\left(P_{i}\right)
$$

has exponent $q^{\ell}$ for $i \in\{1,2, \ldots, s\}$. That is in $\left(^{*}\right)$ we can choose the $h$ 's to be of order $q$. It is clear that for each $i \in\{1, \ldots, s\} H / L_{i}$ acts faithfully on $P_{i}$ so it must be cyclic by $[\mathbf{1}, 3.2 .3]$. Now, since $H$ is homocyclic of exponent $q^{\ell}$,

$$
H=\prod_{i=1}^{t}\left\langle h_{i}\right\rangle
$$

where the order of $h_{i}$ is $q^{\ell}$ for $1 \leqslant i \leqslant t$. Suppose that the exponent of $L_{r}$ is $q^{\ell_{r}}$ for some $r \in\{1,2, \ldots, s\}$ and let $\ell_{r}<\ell$. Then for each $j \in\{1, \ldots, t\}$

$$
\bar{h}_{j}=h_{j} L_{r}
$$

is non trivial in $H / L_{r}$. Next, let $H / L_{r}=\langle\bar{h}\rangle$ so that for $1 \leqslant i, j \leqslant t$

$$
h_{j}=h^{n_{j}} u_{j} \text { and } h_{i}=h^{n_{i}} u_{i} \text { for some } u_{i}, u_{j} \in L_{r} .
$$

In particular $h_{1}=h^{n_{1}} u_{1}$ and $h_{2}=h^{n_{2}} u_{2}$ for some integers $n_{1}$ and $n_{2}$ so that

$$
h_{1}^{n_{2}}=h_{2}^{n_{1}} u \text { for some } u \in L_{r} .
$$

Therefore

$$
h_{1}^{n_{2} q^{\ell_{r}}}=h_{2}^{n_{1} q^{\ell_{r}}}=1 \text { since }\left\langle h_{1}\right\rangle \cap\left\langle h_{2}\right\rangle=1
$$

that is

$$
q^{\ell-\ell_{r}} \mid n_{1}
$$

But then this contradicts that $h_{1}$ has order $q^{\ell}$.

The following result is well known, see for instance $[\mathbf{1}, 3.2 .2]$. 
THEOREM 2.9. If $G$ is Abelian and has a faithful irreducible representation then the centre $Z(G)$ is cyclic.

We close this section with the next result which can be found in $[3,3(\mathrm{iv})]$.

Lemma 2.10. Let $G$ be an $A$-group and suppose that $G=G^{\prime} L$. Then

$$
F \cap L=Z(G)
$$

\section{Proof of the main Result}

In this section we shall bring together the ideas in Section 2 to prove Theorem A.

Proposition 3.1. Suppose that $G=W \rtimes H$ is a monolithic A-group with monolith $W$ and $H$ a metabelian $p^{\prime}$-group which acts faithfully on $W$. Suppose also that the exponent of $H_{q}^{\prime}$ is $q^{\ell(q)}$. Then $G$ is a $C S_{n}$ group if and only if for all primes $q$, every element in $H$ of prime order or of order dividing $q^{\ell(q)}$ lies in $F(H)$. Here $W$ is assumed to be a p-group and $F(H)$ denotes the Fitting subgroup of $H$.

Proof: We start by proving that under the hypotheses $G$ is a $C S_{n}$ group. We note that since $H$ is metabelian then (2.7) and (2.1) reduces the proof to showing that

$$
[W, K] \cap C_{W}(h)=1
$$

where $h \in H, K=\langle h\rangle^{\cdot H}$ and $h$ has prime power order. The next thing to note is that (2.3) (1) implies that $H=H^{\prime} L$, where $H^{\prime}$ is the derived subgroup of $H$. Also by (2.10) $F(H) \cap L=Z(H)$ and $Z(H) \cap H^{\prime}=1$ by (2.3) (2), thus

$$
F(H)=Z(H) \times H^{\prime} .
$$

Now if $h \in F(H)$ then $K=\langle h\rangle$ so that $[W, K]=[W, h]$, hence

$$
[W, K] \cap C_{W}(h)=1 \text { by co-prime action }(2.2) \text {. }
$$

So we may assume that $h \in H \backslash F(H)$ and has order a power of $q$. It is clear that $h$ is conjugate to $u v$ for some $u \in H^{\prime}$ and $v \in L$. Let $\ell(q)=\ell$. By hypothesis every element of $H$ of order dividing $q^{\ell}$ is in $F(H)$, so if $o(h)=q^{r}$ then $r>\ell$ and $o(v)=q^{r}$ while $o(u) \leqslant q^{\ell}$ since $q^{\ell}$ is the exponent of $H^{\prime}$. Now let $s=r-\ell$ then

$$
1 \neq v^{q^{s}} \in Z(H) \text { since } h^{q^{s}} \text { has order } q^{\ell} .
$$

This means that for some $t, \ell \leqslant t<r$

$$
1 \neq h^{q^{t}}=v^{q^{t}} \in Z(H)
$$


But then

$$
C_{W}(h) \leqslant C_{W}\left(h^{q^{t}}\right)=C_{W}\left(v^{q^{t}}\right)=1
$$

since $C_{W}\left(v^{q^{t}}\right)$ is an $H$-module and the action of $H$ is irreducible and faithful.

If $\ell(q)=0$, that is if $H^{\prime}$ has no Sylow $q$-subgroup, and $h$ is an element in $H$ of order $q^{\alpha}$, then $h$ is conjugate to some element $v \in L$. Now $v_{1}=v^{q^{\alpha-1}}$ is an element of prime order $q$ so it must be in $F(H)$. But then

$$
v_{1} \in F(H) \cap L=Z(H)
$$

and again

$$
C_{W}(h) \leqslant C_{W}\left(v_{1}\right)=1 .
$$

Conversely, we have to show that if $G \in C S_{n}$ and $H_{q}^{\prime}$ has exponent $q^{\ell(q)}$ for cach prime $q$, then every element of $H$ of prime order or of order dividing $q^{\ell(q)}$ lies in $F(H)$. Now, by (2.5) $F(H)$ contains all elements of $H$ of prime order. So we may suppose that $h \in H$ and $o(h) \mid q^{\ell(q)}$, say $o(h)=q^{t(q)}$. Then $h$ is conjugate to an element $u v$ for some $u \in H_{q}^{\prime}$ and $v \in L$ such that $v^{q^{\ell(q)}}=1$ and $v \notin Z(H)$.

Let $x \in H_{q}^{\prime}$ be an element of order $q^{t(q)}$. Then

$$
\langle x\rangle \cap\langle v\rangle \leqslant H^{\prime} \cap L=1
$$

so that $A=\langle x\rangle \times\langle v\rangle$ is a homocyclic subgroup of $H$ of exponent $q^{t(q)}$. Now $W$ and $A$ satisfy the hypothesis of (2.8). This means that $W$ is generated by the centralisers of the cyclic subgroups of $A$ of order $q^{t(q)}$. But these cyclic subgroups are exactly $\left\langle x^{v^{j}}\right\rangle$ and $\left\langle v x^{i}\right\rangle$ for $0 \leqslant i, j<q^{t(q)}-1$. Now (2.6) says that $C_{W}\left(v x^{i}\right)=1$ for $0 \leqslant i \leqslant q^{t(q)}-1$. Since $v x^{i} \notin F(H)$. We next let $\left\langle v^{\alpha}\right\rangle=\langle v\rangle \cap F(H)$. Since $F(H)$ contains all elements of $H$ of prime order we know that $1<\alpha \leqslant q^{t(q)-1}$. But for $1 \leqslant \beta<\alpha, v^{\beta} \notin F(H)$ so that $C_{W}\left(x v^{\beta}\right)=1$. So we are left with $\langle x\rangle$ and $\left\langle x v^{j}\right\rangle, \alpha \leqslant j \leqslant q^{t(q)-1}$ the centralisers of which generate $W$. Let $n=q^{t(q)}-\alpha$. Then $C_{W}(x)$ and $C_{W}\left(x^{v^{j}}\right)$ are all contained in $C_{W}\left(x^{n}\right)$, since $\left(x^{v^{j}}\right)^{n}=x^{n}$ because $\left(v^{j}\right)^{n}=1$. Thus $C_{W}\left(x^{n}\right)=W$ and this contradicts the faithful action of $H$ on $W$.

We now come to the proof of Theorem A. We begin by assuming that $G \in C S_{n}$ and show that (1), (2) and (3) hold.

We observe that by (2.4) the monolith $W$ of $G$ is elementary Abelian for some prime $p$ and the Fitting subgroup $F(G)$ is a homocyclic $p$-group. Hence $F(G)=$ $O_{p}(G)=P$. Also $G=P \rtimes H$ where $H$ is a $p^{\prime}$-group. Now since $G$ has nilpotent length three, $H$ has nilpotent length two and is therefore metabelian. Next, because $G$ 
is soluble, $C_{G}(P) \leqslant P$ by $[\mathbf{5}, 7.67]$. In fact $C_{G}(P)=P$ since $P$ is Abelian, therefore $C_{H}(P)=1$.

The next thing to note is that

$$
W \leqslant \Omega_{1}(P)=P_{1}
$$

and the action of $H$ on $P_{1}$ is completely reducible. But $W$ is the monolith so we must have $W=P$. The action of $H$ on $W$ is clearly irreducible, so to finish proving (1) we only need to show that it is also faithful.

Let $p^{n}$ be the exponent of $P$ and suppose that $C_{W}(h)=W$ for some $h \in H$. Then

$$
[P, h, \ldots, h]=1 \text { ( } h \text { appearing } n \text {-times })
$$

since $h$ stabilises the series

$$
1=P_{0}<P_{1}<P_{2}<\ldots<P_{n}=P \text { where } P_{i}=\Omega_{i}(P)
$$

also

$$
P_{i+1} / P_{i} \simeq \Omega_{q}(P) \text { for } 0<i \leqslant n-1 .
$$

But

$$
[P, h, \ldots, h]=[P, h] \text { by co-prime action. }
$$

Thus $C_{P}(h)=P$ and hence $h=1$ since $C_{H}(P)=1$.

We next go to (2). By (2.3) (1) $H=M L$ and clearly $\left[M_{q}, L_{q}\right]=1$ since $H$ is an $A$-group. Also $F \cap L=Z(H)$ and $F=M \times Z(H)$ as we have seen in the proof of (3.1). Now, by (2.5) $F$ contains all elements of $H$ of prime order, and since $L$ is non trivial because $G$ has nilpotent length three we must have $F \cap L \neq 1$. Hence $Z(H) \neq 1$ and every element of $L$ of prime order is in $Z(H)$.

To see that $L$ is cyclic, we first note that $H$ has a faithful irreducible module, so $Z(H)$ has a faithful irreducible representation and by $(2.9), Z(H)$ must by cyclic. But this implies that the subgroup of $L$ generated by all the elements of $L$ of prime order is cyclic. So $L$ itself has to be cyclic.

What remains in the proof of $(2)$ is to show that $[M, L]=M$. But this is clear since

$$
M=H^{\prime}=[M L, M L]=[M, L]
$$

because $M^{\prime}=L^{\prime}=1$.

To finish the first part of the proof, it only remains to show that (3) holds. But parts (1) and (2)(c) imply that (3.1) can be applied and this gives the required result. 
Conversely, we assume that $P, M$ and $L$ are groups satisfying (1), (2) and (3), then we have to show that $G$ as given is a monolithic $A$-group of nilpotent length three in $C S_{n}$.

It is clear that (1) and (2)(a) imply that $G$ is a monolithic $A$-group of nilpotent length three. We next observe that by (2.7) $H$ is a $C S_{n}$ group since it is metabelian. At this point we can apply (2.1) to see that what remains of the proof is to show that if $h \in H$ is a $p^{\prime}$-element of, say, prime $q$ power order then

$$
\left[P,\langle h\rangle^{\cdot * H}\right] \cap C_{P}(h)=1 .
$$

Now, if $h \in F$ then since $F$ is Abelian and normal in $H$ we have $\langle h\rangle^{\cdot H}=\langle h\rangle$ and $\left(^{*}\right)$ holds by co-prime action. So we may assume that $h \notin F$. We next note that $h$ acts fixed point freely on $W$ if and only if it does so on $P$. This is because $W=\Omega_{1}(P)$ and $P$ is homocyclic. By (2.6) for $\left(^{*}\right)$ to hold it is enough to show that $h$ acts fixed point freely on $W$.

Next we note that $h$ is conjugate to $y x$, an element in $M_{q} L_{q}$ which is a Sylow $q$ subgroup of $H$, for some $y \in M_{q}$ and $x \in L_{q}$. Also $h$ acts fixed point freely on $W$ if and only if every conjugate of $h$ behaves in the same way. This is because

$$
[W, h]=W \Leftrightarrow\left[W, h^{g}=W^{g} \Leftrightarrow\left[W, h^{g}\right]=W \forall g \in H .\right.
$$

So it is enough to deal with $y x$. Let $q^{t}$ be the order of $y$ for some $t \leqslant \ell(q)$, the exponent of $M_{q}$ being $q^{\ell(q)}$. Then

$$
(y x)^{q^{t}}=x^{q^{t}}
$$

and if $(y x)^{q^{t}} \neq 1$ then some power of $x^{q^{t}}$ has order $q$, a prime, so it lies in $Z(H)$ by (2)(c) and so it acts fixed point freely on $W$. If on the other hand

$$
(y x)^{q^{t}}=1 \text { then } x^{q^{t}}=1
$$

and $x$ has order dividing $q^{\ell(q)}$ and by (3), $x \in Z(x)$. Thus $y x \in F$ and this case has been dealt with.

\section{Conclusion}

Theorem A gave a rather detailed description of those $A$-groups of nilpotent length three in $C S_{n}$. One would have wanted to have a similar understanding of groups with higher nilpotent lengths. But as the nilpotent length grows the number of subgroups to be dealt with increases and complications will set in.

We know that $A$-groups split over each of their subgroups in the derived series [6], it will be interesting to find what happens inside those $A$-groups in $C S_{n}$, for nilpotent length $n$ greater than three. It is clear that the monolithic ones should again be our main target. This suggests the next logical step. That is to get a good hold on the monolithic $A$-groups in $C S_{n}$ of any nilpotent length $n$. 


\section{REFERENCES}

[1] D. Gorenstein, Finite groups, (second edition) (Chelsea Pub. Comp., New York, 1980).

[2] U.M. Makarfi, 'A class of finite groups satisfying certain subnormal conditions', Afrika Mat. 3 (1991), 65-82.

[3] U.M. Makarfi, 'A theorem on A-groups', (ICTP, Trieste, internal report IC/95/417).

[4] U.M. Makarfi, 'Cyclical subnormal separation in A-groups', (ICTP, Trieste, internal report IC/95/418).

[5] J.S. Rose, A course on group theory (Cambridge University Press, Cambridge, 1978).

[6] D.R. Taunt, 'On A-groups', Proc. Cambridge Phil. Soc. 45 (1949), 24-42.

Department of Mathematics

Ahmadu Bello University

Samaru, Zaria

Kaduna State

Nigeria 